\title{
Correction to: Dietary Interventions and Multiple Sclerosis
}

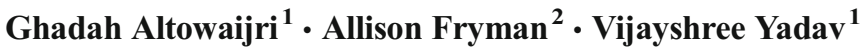

Published online: 17 October 2017

(C) Springer Science+Business Media, LLC 2017

\section{Correction to: Curr Neurol Neurosci Rep (2017) 17:28}

https://doi.org/10.1007/s11910-017-0732-3

The original version of this article contains an error in the second sentence of the second paragraph of the Paleolithic Diet section. The sentence reading "The multimodal approach included diet, massage, acupuncture, and meditation." should be corrected to "The multimodal approach included diet, massage, strengthening exercises, electrical stimulation of the muscles, and meditation."

The online version of the original article can be found at https://doi.org/ 10.1007/s11910-017-0732-3.

\section{Vijayshree Yadav}

yadavv@ohsu.edu

1 Department of Neurology, Oregon Health \& Science University, 3181 S.W. Sam Jackson Park Rd, Portland, OR 97239, USA

2 Department of Veterans Affairs Medical Center, 3710 SW US Veterans Hospital Rd, Portland, OR 97239, USA 